patient himself or in a passive manner by the examiner. Auscultation of the shoulder-joint is also easy, and the sounds produced during rotatory movements, as well as during elevation and depression of the arm, may be alike studied. Nor is any serious difficulty met with in the application of the method to any of the larger joints, such as the elbow, wrist, or ankle. Even small articulations, such as the temporo-maxillary or the carpo-metacarpal joint of the thumb, admit of examination with the stethoscope.

I cannot pretend to give in this paper any complete or adequate account of the sounds heard in moving joints, nor of their diagnostic significance, for I can lay no claim to the knowledge required for the production of such an account. My aim is rather to direct attention to the value of this clinical method, and to stimulate others to employ it, in the hope that the accumulated experience of many observers may lead in time to a just appreciation of the meaning of the sounds and an accurate differentiation of their several varieties. Our knowledge of the meaning of sounds heard in auscultation of the chest has been built up by comparison of clinical observations with post-mortem findings, but the qualities of the sounds are in most instances indicative of the method of their production. Thus a perieardial friction sound strongly suggests its origin, and when we hear sibilus, the rush of air through narrowed passages is clearly indicated. Again from the time and distribution of a cardiac murmur the seat and nature of a valvular lesion may be inferred, and the inference drawn during life has been so often confirmed by examination after death that there is no longer any room for doubt as to interpretation, yet experience shows that even skilled observers sometimes are misled by clinical signs.

In the case of joints the opportunities of confirming our findings by actual examination of the parts are few, and therefore the acquisition of certain knowledge as to the meaning of articular sounds must be mainly a matter of clinical observation, checked by such information as $\mathrm{X}$ ray pictures afford-information which must, in the nature of things, chiefly relate to the condition of the bony structure. When examined with the stethoscope a normal joint of a young subject is silent. It is true that loud reports are sometimes produced in such joints, and can be produced by some persons at will. Such sounds have no morbid significance, and may be compared to the click produced by lifting a ball from a closely fitting socket; in other words, they are vacuum sounds.

In the joints of subjects past middle life abnormal sounds are often heard, even though the patient makes no complaint of his articulations, and is conscious neither of pain nor stiffness. They may be ascribed to the changes due to wear and tear, but experience teaches that in cases of incipient osteo-arthritis aknormal sounds may precede all subjective sensations. Thus when one knee is alone complained of, its fellow may exhibit well-marked auscultatory signs. On the other hand, silence of a joint is by no means a sure indication of health. In cases of synovitis, in which the articular surfaces are held apart by effused fluid, no sounds are heard, as a rule, but when the effusion has been absorbed a pronounced crackle becomes audible at any part to which the stethoscope can be applied, and this sound, to which the name of synovial crackle may be applied, is one of the commonest of morbid sounds heard in joints. Long after the attack of synovitis is past, especially in cases in which the joint which was its seat is apt to give trouble after any unusual use or strain, the synovial crackle may still be audible, and, as far as can be judged on clinical grounds alone, it would appear to indicate a swollen condition of the synovial membrane. In its most characteristic form it resembles closely the crackle heard when the stethoscope is applied to a region which is the seat of surgical emphysema.

It is not only when effusion is present that diseased joints are silent. A very limited experience of auscultation of tubercular joints leads me to believe that these are frequently or even usually silent, and the same is also true of joints affected by the more acute forms of rheumatoid arthritis, although in the later stages the synovial crackle is usually beard in them. This is in accord with the scanty post-mortem evidence available as to the condition of the joints in the acute stage of the disease in question, which tends to show that the joints themselves are at first but little implicated and that the inflammatory swelling is mainly periarticular.
The knee-joints of patients in or past middle life, and especially of women about the period of the menopause, are often the seats of pain and stiffness, which is especially felt in kneeling, or in going up and down stairs, and for which medical advice is sought. In such joints a peculiar abnormal sound is heard, especially well when the stethoscope is applied on either side of the patella. In characteristic cases this is rather a scrunch than a crackle, and has a wholly different character from the synovial sound already described. The character of the scrunch suggests abrasion of cartilages rather than synovial swelling, and radiograms of the articulation in which it is heard usually show slight but distinct evidences of osteo-arthritic changes. I am strongly inclined to think that this scrunch is an early and important sign of osteo-arthritis. It must be mentioned, however, that cases occur in which it is difficult to decide to which class the sounds heard should be assigned, and it is more than probable that in many instances the synovial crackle and osteoarthritic scrunch coexist.

It should be mentioned that I have here employed the words rheumatoid and osteo-arthritis to designate distinct morbid conditions, which I believe to be manifestations of two distinct diseases, and that $I$ believe the osteo-arthritic scrunch to be a physical sign of that process which results in fibrillation and erosion of cartilages and the formation of chondrophytes and osteophytes.

There is another articular sound which is much less commonly heard than either of those which have already been referred to, and which can usually be felt as well as heard. It is a rubbing noise which suggests the friction of ill-lubricated surfaces upon each other, and is such as might result from the movement upon each other of eburnated surfaces of bone. However, such an explanation is not tenable in view of the fact that after persisting for a time the friction may wholly disappear, and it is in the more advanced stages of rheumatoid arthritis, rather than in association with osteoarthritic changes, that this abnormal sound is heard. Into one or other of the above-mentioned categories the morbid sounds heard in joints appear to fall for the most part. Lastly, the friction which may be felt over inflamed tendon sheaths produces a quite characteristic noise, which must be distinguished from those produced within the articulations.

To sum up, it may be claimed for auscultation of joints that this method is capable of affording early warning of troubles in articular structures and sometimes reveals the commencement of disease in joints which to inspection and palpation appear to be normal, and which may even cause no discomfort to their possessor. Again, the method gives promise of help in the differentiation of articular diseases, and in distinguishing between those in which the cartilages and bones are implicated, and those in which the synovial membranes and soft parts around the joints alone suffer. If these claims be justified auscultation of joints does not merely supply information which can be equally well obtained in other ways, but promises to lead to the extension of our clinical knowledge of the conditions in which it is employed. - Chandos-street, W.

\section{TRANSPERITONEAL CYSTOTOMY FOR TUMOUR OF THE BLADDER, WITH REPORT OE A CASE. ${ }^{1}$}

By SETON PRINGLE, M.B. DUB., F.R.C.S. IREL., SURGEON TO MERCER'S HOSPITAL, DUBLIN.

IT has long been recognised that the operative treatment of tumours of the urinary bladder is, to put it mildly, disappointing and unsatisfactory; indeed, such a well-known authority as Casper in his book on "Genito-Urinary Diseases" states that "patients do better and live longer without than with operation."

In considering this subject, we must remember that the vast majority of bladder tumours are epithelial in origin, that the majority of these are carcinomata, and that even the pathologically innocent tumours, the so-called benign papillomata, are often clinically malignant, inasmuch as they recur both in situ and as implantation tumours. We must

Being a paper read before the Surgical Section of the Royal Academy of Medicine in Ireland on Nov, 18th, 1910. 
also, however, remember that the bladder is poorly supplied with Iymphatics, so that the growth of cancerous tumours is slow and that metastases are rarely formed.

At present the surgeon generally chooses the suprapubic route in attacking these tumours, as it is more satisfactory than the infrapubic, perineal, or vaginal route, and does not require the special skill and practice which enable a limited number of operators successfully to use the urethral route in -selected cases. The suprapubic is, and probably will be, the operation of choice in dealing with tumours placed near the apex of the bladder; but, unfortunately, the great majority are situated at the base, and in dealing with these the disadvantages of this route are many and obvious. It is difficult to obtain a proper view of the growth. The hæmorrhage is difficult to control and further obscures the view. In the cases of malignant growths it is almost impossible to define their limits; and if the orifice of either ureter is involved, as it frequently is, the very greatest trouble is found in dealing satisfactorily with the severed ureter. There is also the danger of infecting the prevesical space.

The results also of the suprapubic operations have been most disappointing. Watson (1) has compiled statistics of the result of 662 suprapubic operations for tumour. He found that in 287 performed for benign papilloma there was an immediate mortality of 10 per cent. and a recurrence of 20 per cent. inside three years. In 375 performed for malignant growths the operative mortality was 27 per cent. and the rate of recurrence 63 per cent. inside three years.

The operation of intra- or trans-peritoneal cystotomy, to which I wish to call attention, obviates all the difficulties of technique which I have enumerated above. At the same time it lessens the immediate mortality, and will certainly improve our end-results as it enables us to deal much more radically with the growth. This operation was first performed by Harrington (2) for an intractable case of hæmorrhagic cystitis. He described the operative technique in detail, and is entitled to all the credit of proposing and carrying out an original procedure. As far as one can judge from a study of the literature, this operation was not again performed until March, 1907, when C. H. Mayo (3) employed the technique of Harrington in dealing with a cancer of the base of the bladder. Up to the present, as far as I have been able to ascertain from a fairly extensive study of the literature, some 21 cases of tumour attacked by this route have been reported. C. H. Mayo in 1908 published reports of 5 cases; and Judd (4) has since reported 10 further cases from the Mayo clinic. Of these 15 cases 9 were carcinomata and 6 benign papillomata. There was only one death, and that occurred in a man of 71 , who had half his bladder removed for cancer, and who died from uræmia a week later ; at the post-mortem examination his kidneys were found extensively diseased. Scudder and Davis (5) in 1908 published four cases, three of papillomata and one of benign papillary cystadenoma of the prostate. All these cases recovered. Primrose (6) and Cabot (7) in 1909 reported one case each of cancer successfully dealt with by this route.

On analysing these 21 cases we find that a large part of the bladder was resected several times, a ureter had to be transplanted at least on four occasions, and twice the prostate was also enucleated. In spite of these radical procedures the mortality was less than 5 per cent., which compares with a 10 per cent. mortality in the series of cases collected by Watson, in which the suprapubic route was used and the tumour only removed withont any resection of the bladder wall. As regards the frequency of recurrence, it is yet too early to speak. Up to the time of publication of Judd's paper there had been three recurrences in Mayo's cases, on two of which he successfully re-operated. If we take these two operations and the case now reported, we find the operative mortality further reduced, as there was only one death in 24 operations-i.e., a mortality of $4 \cdot 1$ per cent.

Operation.-After the bladder has been washed out and emptied the patient is placed in the Trendelenburg position. The peritoneum is opened by a long median incision extending for six incines or more up from the pubis. In malignant cases the interior of the abdomen is then carefully inspected, to eliminate the presence of metastases before proceeding further. If these are absent, as they usually are, or if the case is of a benign nature, the intestines are now carefully packed off with gauze. The superior peritoneally covered surface of the bladder is now seized with two forceps and a short median incision made into it. The interior is carefully swabbed out and the incision lengthened as much as necessary. In this way an excellent view is obtained. (Fig. 1.) If the tumour is pedunculated, it is grasped in forceps and removed with the knife or scissors, so that some of the normal mucous and submucous coats is also taken. The raw surface left should then be

\section{FIG. 1.}

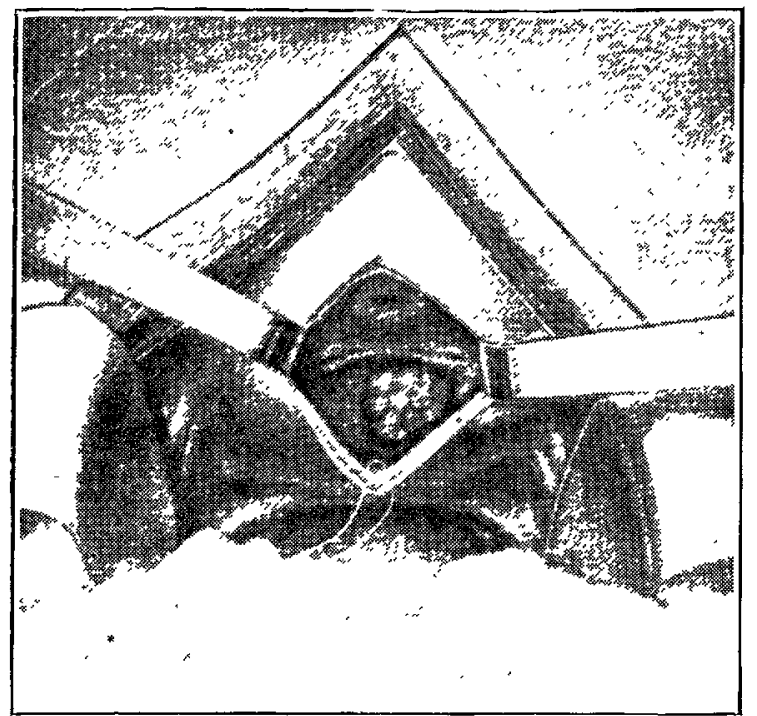

Note the long abdominal incision well retracted, intestines protected by gauze, low posterior incision into bladder, internal meatus, ureteral orifices, tumour, and traction suture. (Scudder, Annals of Surgery.)

cauterised with the Pacquelin cautery, or, if preferred, the whole removal may be performed with the cautery. (Fig. 2.) Sutures will not, as a rule, be required, as the cautery controls the bleeding, and the raw surface may be left to granulate up. In malignant cases the whole thickness of the involved part of the bladder wall, whether covered by peritoneum or not, must be removed, the section being made

FIG. 2.

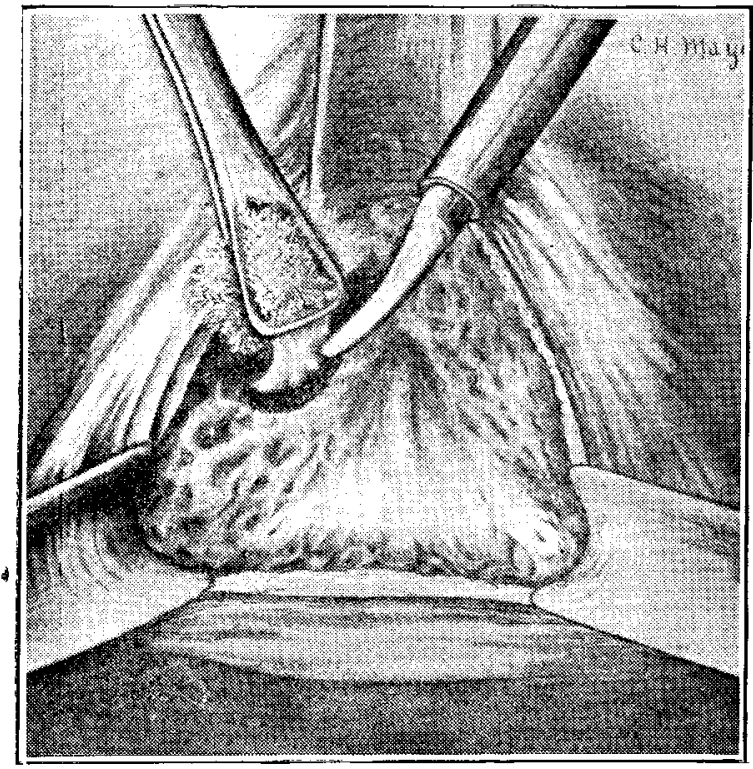

Cautery resection of papilloma of bladder. (Mayo, Annals of Surgery.)

through normal tissue. In some cases as much as half of the bladder wall has been removed, and in others the floor of the space left after removing the tumour has been formed by the fibres of the levator ani. If the ureter is involved in the growth, its orifice is removed en masse with the tumour, and it is divided above the involved portion. Its distal end is then re-implanted into the bladder, either through a separate punctare (Fig. 3) or through the original incision, and fixed there with catgut sutures. On completing the intravesical part of the operation the bladder wall is completely closed by a double row of sutures, ans inner Connell suture of catgut 
(Fig. 4), and an outer peritoneal suture of fine silk (Fig. 5).

As a rule, the abdominal wound may be closed in its entirety, but if there has been any soiling of the peritoneum a drain may be left in for a few days. In a simple case it is not necessary to drain the bladder, as the patient generally passes urine voluntarily and at frequent intervals from the evening of the operation, and if there is any urethral spasm

FIG. 3.

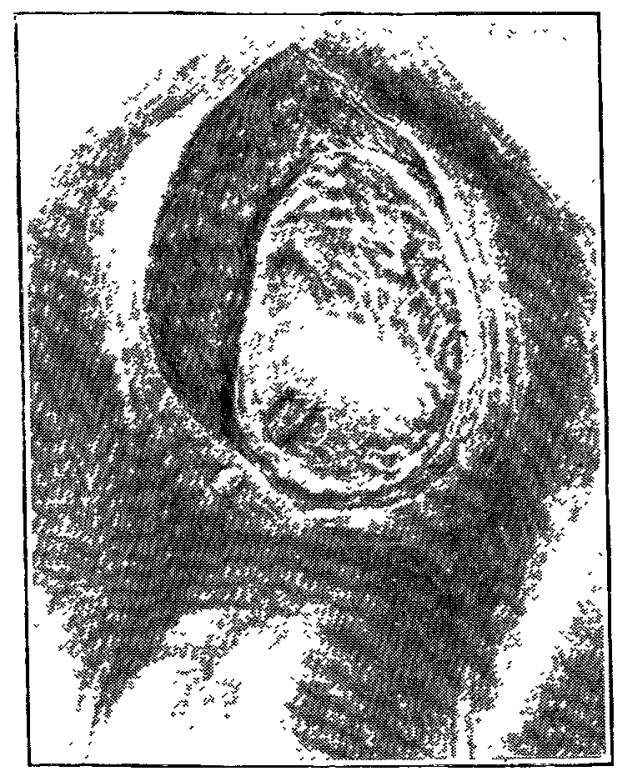

Removal of large amount of bladder with transplantation of ureter. (Mayo, -

a catheter can be frequently passed. Some operators, however, drain these simple cases by means of a catheter in the urethra. In severe cases, as for instance where the prostate has also been removed or a ureter transplanted, it is

FIG. 4.

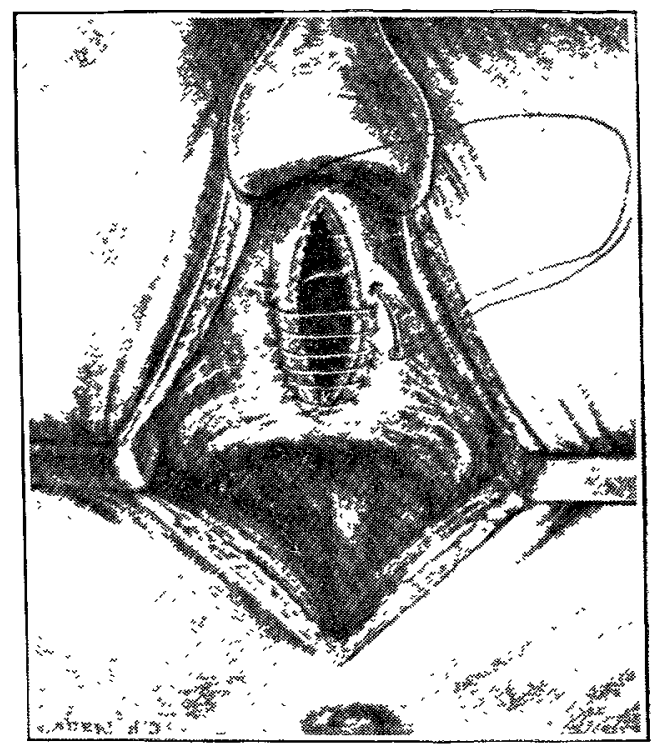

Showing method of closure of bladder incision. First row of sutures. (Mayo, Annals of singery.)

better to drain the bladder suprapubically through an independent incision into the bladder, made after the peritoneal cavity has been closed.

My thanks are due to the publishers of the Annals of Surgery for permission to use the accompanying illustrations.

Report of case. - The patient, a man aged 40, was sent to me by Dr. H. Powell of Nenagh. He complained of frequent attacks of painless hematuria during the past 12 months, each attack lasting several days. With the cystoscope I saw a large papilloma springing from the right side of the bladder, but it was impossible to clearly define its origin, as the floating papillæ, of which the bladder seemed to be full, constantly obscured the view. On June 1st I performed transperitoneal cystotomy as described. The tumour, which was of the size of a hen's egg, was pedunculated, the pedicle springing from the base of the bladder about an inch below and to the right of the right ureteral orifice, which was not interfered with. An excellent view was obtained, and the tumour was easily removed with scissors, a piece of normal mucous membrane being taken with it. The raw area left, which was about an inch square, was then cauterised with a Pacquelin cautery, and one stitch of catgut inserted to control a bleeding point. The bladder was sewn up as described, and was not drained. The abdominal wound was completely closed. The patient made an uninterrupted recovery, the wound healing by first intention. He passed urine voluntarily the evening of the operation, and continued to do so at frequent intervals. The urine was slightly tinged with blood for the first few days. On June 24th I again examined his bladder with the cystoscope, and could see the line of incision soundly healed, while the

FIG. 5 .

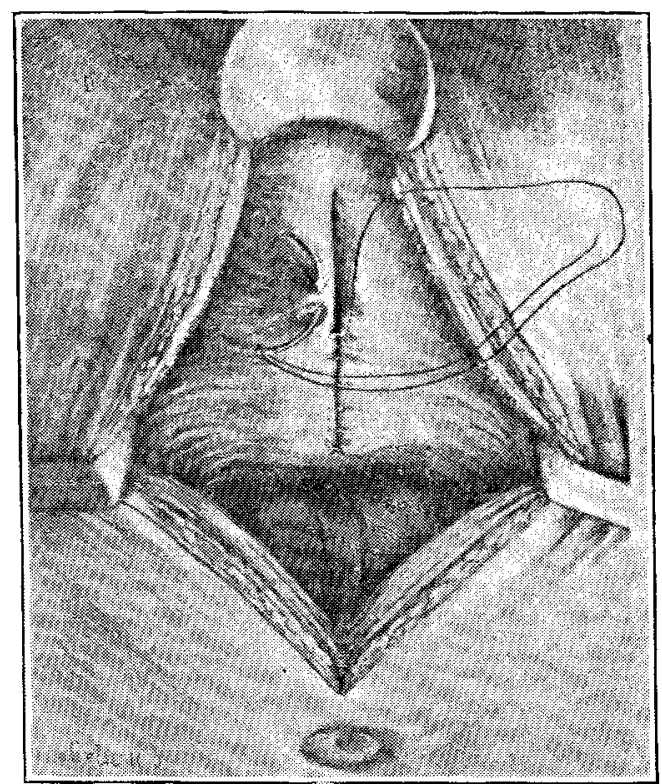

Second row of suture elosing the bladder. (Mayo, Annals of Surgery.)

site of the growth was marked by a small area of granulations. He went home the next day - that is, on the twentysixth day after operation, and was then able to retain his urine for five hours.

Note.-Since writing the above I find that Richardson in 1895 successfully performed transperitoneal cystotomy on two cases for the removal of large calculi on which the bladder had firmly contracted. 2

Bibliography. -1. Watson: Annals of Surgery, December, 1905. 2. Harrington : Ibid., October, 1893. 3. Mayo, C. H. : Ibid., July, 1908. 4. Judd : Journal of the American Medical Association, Dec. 25th, 1909. 5. Scudder and Davis: Annals of Surgery, December, 1908. 6. Primrose: Ibid., December, 1909. 7. Cabot: Transactions of the American Association of Genito-Urinary Surgeons, vol. iv. 1909.

Dublin.

2 Annals of Surgery, vol. xxxiii., p. 132.

King's Colilege (University of London).-A course of four lectures on Recent Progress in Physiological Chemistry will be delivered in the Physiological Laboratory, King's College, London, by Dr. Otto Rosenheim, at 4.30 P.M., on alternate Mondays, beginning Jan. 30th. The subjects dealt with will be Inorganic Substances, Carbohydrates, Fats, Proteins, Lipoids; the Chemistry of Colloids and Enzymes. The lectures are free to all $\mathrm{m}$ ' mbers of King's College, to all students of medicine, to all internal students of the University of London, and to medical men on presentation of their cards 
PUERPERALCONVULSIONS, OR ECLAMPSIA,

AT THE LIVERIOOL WORKHOUSE HOSPITAL.

BY WILLIAM ALEXANDER, M.D.R.U.I., F.R.C.S. ENG., VISITING SLRTEEN.

THERE is no disease where the results of treatment are so unsatisfactory and so uncertain as in puerperal eclampsia, and in few diseases are there so many, so varied, or such opposite methods of treatment. This arises from the imperfect knowledge of the disease that we possess and from the apparent facility with which serious cases rapidly improve, or the rapidity with which apparently favourable cases become worse or unexpectedly prove fatal. Hence the undue advocacy of remedies which have been followed by recovery in apparently hopeless cases by some medical men, and the low opinion of the efficacy of the same remedies by other medical men who have used them in apparently favourable cases that, nevertheless, turned out unsuccessfully.

A feeling of uncertainty is universal amongst medical men when called to treat a case of eclampsia, and a very guarded prognosis is always given. The necessity of judicious antepartum treatment is always insisted on, and no doubt the management of the pregnant woman is of great importance ; but here again we have no certain methods of treatment that will always ward off eclampsia. Many years ago the writer had a share in the treatment of a lady who was attacked by albuminuria and oedema at mid-term. All the resources of medical knowledge were used to ensure the disappearance of the symptoms before delivery, and success, as far as the disappearance of albumin was concerned, was obtained and safety seemed assured. Nevertheless eclampsia set in post partum, and death took place in spite of all our efforts. On April 3rd, 1906, a woman, aged 19, a primipara, presented herself in the maternity ward suffering from oedema and albuminuria, and 7 months pregnant. She was sent to the general ward to be dieted, but went out against advice. We heard afterwards that she had been delivered naturally, after which the odema disappeared.

Miss Aldworth, the sister in charge of the maternity wards, has made out for me a statement of the condition of the urine in the patients admitted there for the past five years. From this statement we find albuminuria present in 57 cases, accompanied by general œdema in 25 cases, accompanied by cedema of the lower extremities in 13 cases, albuminuria without any codema in 19 cases. The quantity of albumin varied from a trace up to 16 grammes. All these eases were delivered safely without any eclampsia, but headaches, blurred vision, stupor, \&c., are recorded in a few cases. Many of them were delivered without any preparation, but where possible salines and simple diet were prescribed, so far as time permitted.

The failure of preparatory hygienic measures to save from eclampsia the lady first referred to, and the impunity with which so many cases were delivered safely under the same apparent conditions, show that with the utmost care we cannot always avoid a catastrophe. Nor do carelessness and indifference always lead to one.

Below are brief notes of 38 cases of eclampsia.

Eclamptic Cases in Lying-in.

CASE 1.-Admitted Oct. 13th, 1903. Aged 25; married; primipara. Condition on admission: Os nearly fully dilated ; trace of albumin in urine. Subsequent course: Fits began during second stage; had three; then chloroform administered and patient delivered with forceps of healthy child. Fits continued after delivery, patient having 11 during the next 12 hours and being semi-comatose between. Treatment: (a) Before delivery-blanket bath. (b) After delivery-(1) Enema; (2) potassium bromide (24 grains) ; (3) later, oxygen inhalation
frequently. Progress : Fits ceased after 12 hours. Became more conscious; headache complained of. Fair quantity of urine passed. Bowels kept well opened with saline purgatives. Made good recovery and discharged in 13 days with still a slight trace of albumin in urine.

CASE 2.-Admitted Dec. 30th, 1904. Aged 25; married; primipara (stillborn) and one miscarriage. A week previous to admission face and hands began to swell. Condition on admission: Unconscious; os not dilated; urine loaded with albumin. Subseguent course: Two tits occurred during first stage; none during second. Natural delivery (child premature, decomposed) One fit during third stage and four during next eight hours. Treatment: (a) Defore delivery : Bath ; enema. (b) After deliverv: (1) Dry cupping o erkidneys ; (2) pilocarpine nitrate (I grain hypodermically four hourly) (3) oxygeninhalation frectuently; (4) vapour bath ; (5) potassium bromide (E4 grains four hourly); (6) pulv. jalapæ co. (1 drachm, repeated in 12 patient continued drowsy. Milk and barley-water by mouth. Urine remained scanty and loaded with albumin for next 24 hours. Next day
urine groatly increased, and less albumin. Recovery uninterrupted. Albumin disappeared from urine.

CASE 3.-Ndmitted Dee. 31st, 1904. Aged 23; single; primipara. Condition on admission: Os dilated to size of sixpenny-piece. Weak pains. Subsequent course: Delay in second stage. Chloroform hours after delivery she had a fit, a second after eight hours, and a thir five hours later. Urine loaded with albumin. Treatment: (1) Chloral (15 grains), potassium bromide (24 grains), latter repeated four hourly ; grain hypodermically); (5) oxygen inhalation. (4) pilocarpine nitrate Grain hypodermically); (5) oxygen inhalation. Progress : Had no more
tits after third. Condition improved rapidly in next few days, urine becoming more copious and containing less albumin. Good recovery. CA.SE 4. -Admitted March 19th, 1905. Aged 23; single; primipara. Fits CAsS 4. - Admitted March 19th, 1905. Aged 23 ; single; primipara. Fits
began seven hours before admission-nine altogether. Condition on admission: Comatose; anasarca. Subseriuent course: Irad 11 fits in admission: Comatose; anasarca. Subsecquent course: Ilad 11 fits in
five- hours. Urine scanty, loaded with albumin. Five hours after five- hours. Urine scanty, loaded with albumin. Five hours after 40 hours; no fits during this time. Patient delivered seven hours after labour began of stillborn, decomposed child. Treatment: (1) Vapour labour began of stillborn, decomposed child. Treatment : (1) Vapour pack; (5) saline enema 1 pint. Progress : After delivery patient conpack; (5) saline enema 1 pint. Progress : After delivery patient con-
tinued comfortable for 60 hours, when she had two slight fits in suceession. A hot pack was given, and no further fits occurred. Urine during next few days inereased in quantity, ancl the albumin decreased. Patient made good recovery.

CASF 5.-Admitted April 9th, 1905. Aged 36; married; V.-para Had lad gastric trouble for 14 days before admission. Fits commenced 13 hours before admission, several occurring. Condition on admission : Semi-comatose. Labour just commenced. Subsequent course : During first stage (which lasted 11 hours) patient had two fits. Second stage two bours and had no fits ; natural delivery; child small, premature. Patient. continued restless and delirious all day. Urine very seanty with trace of albumin. Treatment: (a) Before delivery-(1) Magnesium sulphate (90 grains three hourly); (2) enema; (3) saline injection (four hourly); (4) oxygen frequently. (b) After delivery-Hot pack, repeated after 16 hours, and again 9 hours later. Progress: Had no fits after delivery. Continued fairly well for three days, then became worse, incontinent, dic. Dull, listless. Temperature rose to $103^{\circ} \mathrm{F}$. Died
delivery from stupor and asthma. Necrops not granted.

C.ASE 6.-Admitted Dec. 18th, 1905 . Aged 19; single; primipara. Condition on admission: Face, hands, abdomen, and legs swollen. First stage of labour well advanced. Subsequent course : Six hours after stage of labour well advanced. Subsequent course : Six hours after
admission had a fit. Chloroform administered and patient delivered by admission had a fit. Chloroform administered and patient delivered by
forceps of full-term stillborn child. An hour later had a second fit. forceps of full-term stillborn child. An hour later had a second fit. (1) Enema, bath ; (2) tr. scillæ (24 minims), spir. æth. nit. (24 minims), pot. acetat. 6 grains three hourly ; (3) chloral (60 grains per rectum); (4) oxygen frequently; (5) calomel (5 grains), pulv. jalapæ co. (20 grains); (6) vapour bath. Progress : After second fit continued much
better. Improved daily. Urine rapidly increased and albumin decreased. better. Improved daily.

Uninterrupted recovery. Condition on admission: Slight pains. Os not dilated. Trace of albumin in urine. Subsequent course : Shortly after admission patient had a fit. During first stage (which lasted 12 hours) patient had 20 fits. An hour later delivered naturally of twins, having three fits during second stage. During next four hours she had 11 fits. Became incontinent, cyanosed, unconscious. Treatment: (1) Bath, enema ; (2) calomel (5 grains), pulv. jalapa co. .25 grains); (3) oxygen fre-

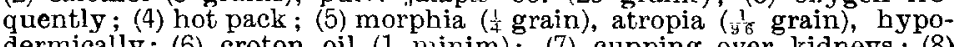
dermically; (6) croton oil (1 minim) ; (7) cupping over kidneys; (8)
vapour bath ; (9) potassium bromide (24 grains), chloral (30 grains per vapour bath;
rectum): (10) venetassium (14 ounces withdrawn); (11) ice-cap to head. Progress: Fits ceased four hours after deliverv, leaving patient unconscious and cyanosed. Given liquor strvchnina hydrochloride ( 3 minims) scious and eyanosed. Given liquor strvchnina hydrochloride (3 minims)
and digitalis (1 50 grain) hypodermically, brandy (1 ounce). Temperature and digitalis (1 50 grain) hypodermically, lrandy (1 ounce). Temperature
rose to $105.4 \circ \mathrm{F}$, so was ice sponged. Saline enema (20 ounces) given. rose to $105^{\circ} 4^{\circ} \mathrm{F}$, so was ice sponged. Saline enema (20 ounces) given.
Three hours later strychnine and digitalis repeated. Breathing now Three hours later strychnine and digitalis repeated. Breathing now difficult; mustard poultice given. Lrine very scanty. Condition
slightly improved during next 12 hours. The patient slowly grew worse and died next day. No necropsy allowed.

CASE 8.-Admitted Oct. 3rd, 1906. Aged 18; single; primipara, Condition on admission: Slight odema of legs; small quantity of
albumin in urine. Os dilated to size of a shilling. Subsequent course : albumin in urine. Os dilated to size of a shilling. Subsequent course: First stage finished nine hours after admission. Two hnurs later delivered naturally of healthy, full-terme child. Labour in all respects seemed normal. One hour after delivery patient had eclamptic fit, followed by
a second and a third at intervals of 15 minutes. Did not regain con a second and a third at intervals of 15 minutes. Did not regain con
sciousness between the fits. Treatment: $(a)$ Before delivery sciousness between the fits. Treatment: (a) Before delivery-
(1) Enema; (2) magnesium sulphate (90 grains, four hourly); (3) milk diet. (b) After delivery - (1) Copious enema, (2) oxygen frequently (3) mist. diuretic. (1 ounce, three hourly). Progress: After the first three fits had passed off the patient became quite conscious and spoke rationally. Three hours after third fit she had a fourth, and then frectuent attacks. Reeovered completely. Went out quite well.

CASE 9.-Admitted Dee. 18th, 1907. Aged 22 years; single; primipara. Condition on admission: Os partially dilated. Urine contained large quantity of albumin. Subsequent progress: Delivered of healthy child three hours after admission; labour normal. Seven hours after delivery patient had an eclamptic fit and two more at intervals of ten minutes; conscious in interval. Transferred to surgical wards where she had five more fits in one and a half hours. Atter this she remained comatose and died 28 bours after delivery. Necropsy: Widema of lungs.

CASE 10.-Admitted Jan. 19th, 1908. Aged 20; single; primipara. Condition on admission: Feet, legs, and abdomen cedematous; face puffy and cyanosed. Os partially dilated. Urine contained small delivered naturally of healthy child. Seven hours after delivery patient had an eclamptic fit, and cluing next ten hours had seven more. Treatment: (1) Copious enema saponis ; (2) magnesium sulphate (1 $\frac{1}{2}$ drachms hourly); (3) hot pack (repeated in four hours); (7) morphia (1 grain hypodermically); (5) ice cap to head ; (6) potassium bromide (12 grains);

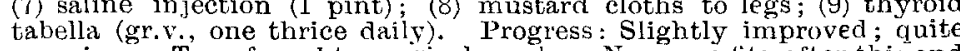
conscious. Transferred to surgical warits. No more tits after this and no further treatment. Uninterrupted recovery. 